\title{
Evaluation of Streptococcus Mutans Biofilm Retention by Three Different Types of Ligations on Orthodontic Brackets: An In- Vitro Scanning Electron Microscopic Study
}

\author{
Rohit Pani Shankar. $\mathrm{K}^{1}$, Krishnan Mahalakshmi ${ }^{2}$, R.V.Murali ${ }^{3}$, \\ Kesavaram Padmavathy ${ }^{4}$, Saravanan Balasubramanian ${ }^{5}$ \\ ${ }^{1}$ (Ex-Post Graduate, Department of Orthodontics and Dentofacial Orthopaedics, Sree Balaji Dental College \& \\ Hospital / Bharath University, India) \\ ${ }^{2}$ (Professor, Department of Microbiology, Sree Balaji Dental College \& Hospital / Bharath University, India) \\ ${ }_{3}^{3}$ (Professor \& Head, Department of Orthodontics and Dentofacial Orthopaedics, Sree Balaji Dental College \& \\ Hospital / Bharath University, India) \\ ${ }^{4}$ (Assistant Professor, Department of Microbiology, Sree Balaji Dental College \& Hospital / Bharath \\ University, India) \\ ${ }^{5}$ (Reader, Department of Orthodontics and Dentofacial Orthopaedics, Sree Balaji Dental College \& Hospital/ \\ Bharath University, India)
}

\begin{abstract}
Introduction - The aim of the study was to evaluate Streptococcus mutansMTCC 890 biofilm retention on the orthodontic brackets with 3 different techniques of ligation using scanning electron microscopic analysis. Methods and Materials - The extracted premolars for orthodontic purpose were taken and were stored in normal saline solution. The 60 extracted premolars were divided into 3 groups:Group - A - Conventional PEA metal brackets with .022 in slot with elastomeric or rubber module $(n=20)$, Group $-B-$ Conventional PEA metal brackets with .022 in slot with steel wire ligatures $(n=20)$ and Group $-C-$ Self ligating brackets $(S L B)$ with .022 in slot for straight arch technique $(n=20)$. The tooth samples were immersed in Brain-Heart Infusion Broth (BHIB) containing S.mutans and incubated aerobically for 72 hours at $37^{\circ} \mathrm{C}$ to allow biofilm formation. The formation of biofilm and its retention to the brackets was confirmed by Scanning Electron Microscopy $(S E M)$. Results - The SEM images showed significant statistical difference $(p<0.05)$ in biofilm retention between the three groups of ligating method. Brackets ligated with elastomeric modules and SLB had more biofilm retention when compared to steel wire ligature group. Conclusion - Fixed orthodontic appliances significantly increase the retention of biofilm regardless the type of bracket system chosen. The result of the present study indicates that steel ligature had least amount of biofilm retention when compared to elastomeric module and self-ligating bracket.
\end{abstract}

Keywords: Ligation techniques, PEA, SEM, SLB, S.mutans

\section{Introduction}

Malocclusions are one of the most common oral health problems, and are associated with a number of complications. Patients who seek orthodontic treatment to correct dental malocclusions often have difficulty in keeping their teeth clean due to crowding. After placement of fixed orthodontic appliances, these patients are at higher risk for caries due to increased plaque retention around brackets and bands. Orthodontic treatment with fixed appliances often interferes with oral hygiene and may thus jeopardize dental health. Streptococcus mutanshave been found to be elevated in patients after placement of orthodontic appliances ${ }^{[1,2]}$. There is a rich ecosystem in the oral cavity, with a countless number of microorganisms. Although both periodontal disease and dental caries are considered multifactorial diseases, the bacteria in the dental plaque are the main factor in their onset and progression. Despite recent advances in orthodontic materials, there has been no decrease in the prevalence of enamel demineralization near the bracket-tooth junction.

The different components of the fixed orthodontic system may contribute to a shift in the balance of the oral ecology creating an "ecological stress". Many studies ${ }^{[3-15]}$ have evaluated the effect of fixed orthodontic appliances on microbial flora and periodontal status however, only a few studies ${ }^{[16-20]}$ have evaluated the type of ligation as an additional factor. The wires are secured/ ligated to the bracket by three methods; (i) ligation with steel wires, (ii) ligation with elastomeric ligatures and (iii) self-ligating brackets. Many studies documented; suggest that the elastomeric ligatures increase the retention of dental biofilm compared with the two other methods. In this study we used scanning electron microscopic images for evaluating biofilm retention to the orthodontic brackets and ligation methods as it is a faster and convenient technique in screening the microbial samples. 
The aim of the present study is to evaluate Streptococcus mutansMTCC 890 biofilm retention on the orthodontic brackets with 3 different techniques of ligation. The following were the objectives of the study: Preparation of orthodontic brackets with 3 different techniques of ligation and comparison. Formation of Streptococcus mutansMTCC 890 biofilm on the orthodontic brackets.Analysis of biofilm formation on the orthodontic brackets by Scanning electron microscope.

\section{II.1 Collection of samples}

\section{Materials and Methods}

The 60 extracted premolars for orthodontic purpose were taken and were stored in normal saline solution and were divided into 3 groups:

Group - A - (Fig 1.A) Conventional PEA metal brackets with .022 in slot with elastomeric or rubber module $(\mathrm{n}=20)$

Group -B - (Fig 1.B) Conventional PEA metal brackets with .022 in slot with steel wire ligatures $(n=20)$

Group - C - 20 (Fig 1.C) Self ligating brackets with .022 in slot for straight arch technique respectively (Victory Series $^{\mathrm{TM}}$, MBT $^{\mathrm{TM}}$, Unitek 3M corporation, Monrovia, California) with .022 in slot and the self-ligating brackets were Smart Clip-SL3 ${ }^{\mathrm{TM}}$ (Unitek 3M corporation, Monrovia, California) .022 in slot.

The elastomeric ligatures were Ormco ${ }^{\mathrm{TM}}$ of size .120 in and the steel ligatures (Leone) of 0.010 in were used. All the brackets used were first premolar brackets.

\section{II.2 Preparation of tooth samples}

The extracted premolar tooth samples were stored in normal saline solution until the study was performed. All the tooth samples were disinfected before the study by immersion in $1 \mathrm{~mL}$ of $70 \%$ ethanol for 30 minutes. The conventional technique of etching with $37 \%$ orthophosporic acid was done and the bonding agent (3M Unitek ${ }^{\mathrm{TM}}$ Transbond $^{\mathrm{TM}}$ XT Light Cure Adhesive Primer) was applied and light cured for 20 seconds. The brackets were bonded to the tooth samples using bracket tweezer and bracket bonding adhesive (3M Unitek $^{\mathrm{TM}}{ }^{\text {Transbond }}{ }^{\mathrm{TM}} \mathrm{XT}$ Light Cure Adhesive Paste). The flash material generated after bracket placement was removed with orthodontic probe. The tooth samples with brackets placed were light cured for 20 seconds using LED light cure unit $\left(\mathrm{Mectron}^{\mathrm{TM}}\right)$.

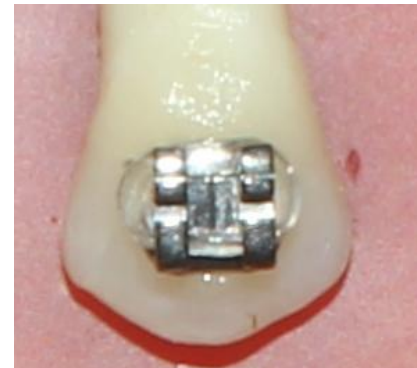

A

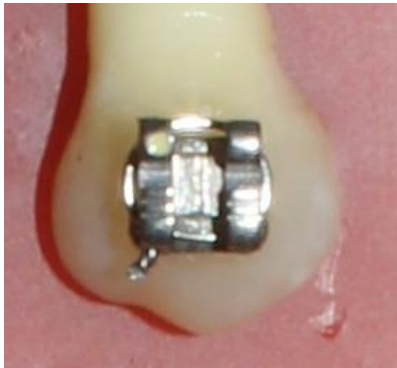

B

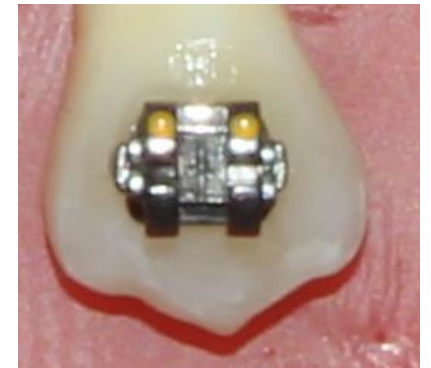

$\mathrm{C}$

Figure 1 - Premolar teeth bonded with bracket and Ligated with A- Elastomeric module, B - Steel Ligature and C - Self-Ligating bracket.

\section{II.3 Procedure}

Fifteen ml of Brain-Heart Infusion Broth was dispensed in each screw capped bottles. The orthodontic brackets along with the tooth sample were placed in the bottles with BHIB and sterilized by autoclaving at $121^{\circ} \mathrm{C}$, $15 \mathrm{lbs}$ pressure for 15 minutes. To the sterile bottles with BHIB $100 \mu \mathrm{l}$ of the Streptococcus mutans MTCC 890 culture inoculum $(1.5 \times 108$ colony forming units $/ \mathrm{ml})$ was added and incubated aerobically for 72 hours at $37^{\circ} \mathrm{C}$ with shaking $(100 \mathrm{rpm})$ to allow biofilm formation. The culture media (BHIB) was replaced every 24 hours. The formation of biofilm and its retention to the brackets was confirmed by Scanning Electron Microscopy (Hitachi S $-3400 \mathrm{~N}$ at ACC voltage of $0.3-30 \mathrm{KV}$ ). The above procedure was performed for the three groups. The orthodontic brackets along with the tooth sample was removed from the BHIB and irrigated with saline solution to remove the unbound cells. All the samples were fixed with $10 \%$ formalin solution and subjected to SEM analysis. The samples were sputter-coated with gold prior to SEM analysis. The samples were analysed blindly by two persons and the results were tabulated.

\section{II.4 Statistical Analysis}

Results obtained were tabulated for each group individually and t-Test was performed and p-value $\leq$ 0.05 was considered statistically significant. To obtain the significance between three groups One Way Analysis 
of Variance (ANOVA) was done and results were graphically represented. The software used for statistical analysis is SPSS version 18.0.

\section{Results}

The Scanning electron microscopic images were visually examined at 20X magnification (Fig. 2 A, B, C) and scored based upon the amount of biofilm present by two observers familiar with SEM analysis in a double - blind manner. The biofilm retention on the three types of ligations on orthodontic brackets were scored and categorized on five different parts of the brackets [Bracket slot Interface,Mesio-gingival wing, Mesio-incisal wing, Disto-gingival wing and Disto-incisal wing]. The scores were given as: $(-)$, sparse $(+)$ and extensive $(++)$.The biofilm retention of the 20 samples belonging to elastomeric module (Group-A) had higher amount compared to that of Group-C (Self ligation locks). While Group - B (steel ligatures) had the least amount of biofilm retention.

The over-all mean of the three groups taken are Group-A (1.12), Group-B (0.25) and Group-C (1.11) (Table -1$)$. When all the three groups were compared together there was statistically significant difference observed between Group - A (Elastomeric module) and Group - B (Steel ligature) $(p<0.001)$. Similarly statistically significant difference was observed between Group - B (Steel ligature) and Group - C (Self ligation lock) ( $\mathrm{p}<0.001)$. Conversely no difference was observed between Group - A (Elastomeric module) and Group $\mathrm{C}$ (Self ligation lock) $(\mathrm{p}=0.961)$. One Way Analysis of Variance was done to find out the significance between the groups (Table - 2). The mean value for Group - A and Group - C were high compared to Group - B. Among the three groups the mean value for Group - B was low. One Way Analysis of Variance shows statistical significant difference in Group - B.

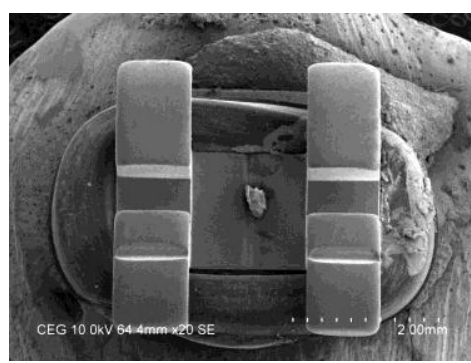

A

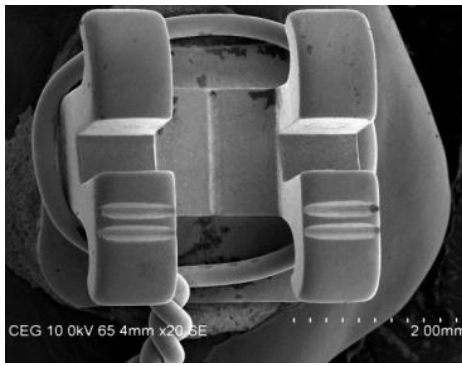

B

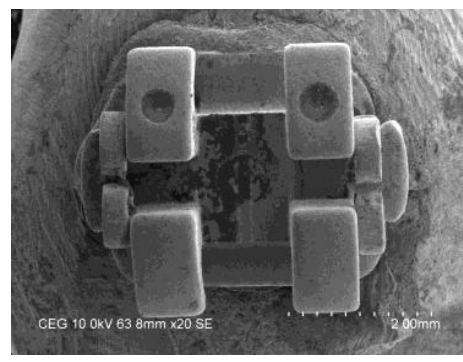

C

Figure 2 - Scanning Electron Microscopic images of A - Elastometic module, B -Steel ligature wire and C- Selfligating bracket

\begin{tabular}{|l|l|l|l|}
\hline \multirow{2}{*}{ Group } & \multirow{2}{*}{$\mathrm{N}$} & \multicolumn{2}{|l|}{ Subset for alpha $=.05$} \\
\cline { 3 - 4 } & & 1 & 2 \\
\hline Ligature wire & 20 & .2500 & \\
\hline Self-ligation lock & 20 & & 1.1100 \\
\hline Rubber Module & 20 & & 1.1200 \\
\hline
\end{tabular}

Table - 1 Overall mean for 3 groups

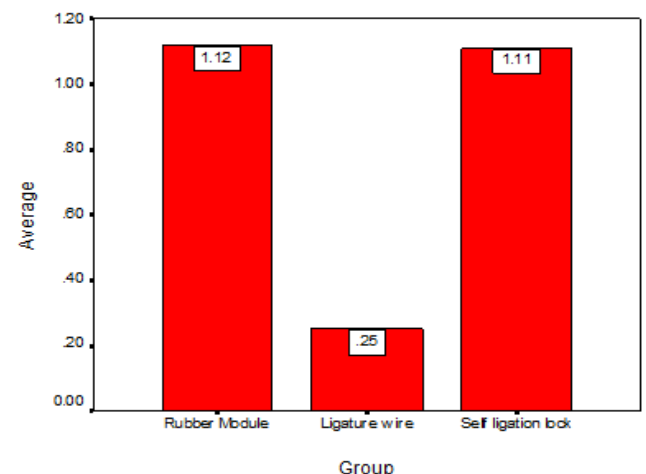

Graph - 1 Graphical representation comparing the biofilm retention between the three groups 


\begin{tabular}{|c|c|c|c|c|c|c|c|c|}
\hline & \multicolumn{6}{|c|}{ Group } & \multirow{2}{*}{\multicolumn{2}{|c|}{ One way ANOVA }} \\
\hline & \multicolumn{2}{|c|}{ Rubber Module } & \multicolumn{2}{|c|}{ Ligature wire } & \multicolumn{2}{|c|}{ Self-ligation lock } & & \\
\hline & Mean & SD & Mean & SD & Mean & SD & \multirow{2}{*}{16.399} & \multirow{2}{*}{.000} \\
\hline Average & 1.120 & .521 & .250 & .274 & 1.110 & .752 & & \\
\hline
\end{tabular}

\section{Discussion}

Patients with orthodontic appliances apart from providing new retention areas of bacterial colonization will also experience oral ecologic changes such as low salivary $\mathrm{pH}$, increased retention of food particles which may lead to increase in levels of salivary S.mutans ${ }^{[19,21]}$. It is unfortunate that fixed appliance orthodontic treatment causes iatrogenic decalcification of tooth enamel and development of visible white spot lesions.

The aim of the study was to evaluate in-vitro the S. mutansMTCC 890 biofilm retention on the orthodontic brackets with three different types of ligation techniques using the images obtained to analyse from SEM. Brain-Heart infusion broth medium was used and the tooth samples with brackets were suspend into the BHIB media. This media helps in growth of fastidious organisms like S.mutans. Sheep blood is preferred for the study as the human blood contains many anti-bacterial substances which may inhibit the growth of S.mutans.

Many studies have assessed the effect of orthodontic appliances on microbial flora with reference to S.mutans levels ${ }^{[1,3,22,23]}$ but the present study evaluated the biofilm retention by three different types of ligation on orthodontic brackets.

Forsberg et al ${ }^{[16]}$ first demonstrated the method of ligation of 2 different types of ligation on fixed appliances with steel wires and elastomeric rings with its effect to microbial colonization around them. They found that lateral incisors that were attached to archwire with E-rings had greater number of microorganisms to that attached to steel wires. They also recommended the use of steel wires in patients with bad to poor oral hygiene as E-rings can increase the bacterial counts on tooth surface and cause dental caries and gingivitis.

On the other hand Turkkahraman et al ${ }^{[6]}$ presented an in-vivo study that revealed, elastomeric ligature had higher number of bacterial organisms to steel ligatures but the differences between them were not statistically significant and could be ignored. With regard to present study, it aimed at evaluating three different ligating techniques instead of two and also used premolar teeth for the study.

Sukontapatipark et al ${ }^{[24]}$ employed 2 methods of ligation with E-rings and steel ligature and used SEM to evaluate the bacterial colonization with fixed metal appliances in premolar teeth and reported that both elastomeric rings and steel ligature had bacterial colonies adhesion and moreover reported that the method of ligation did not seem to influence on microorganisms on either composite resin or enamel surfaces.

Wilson et al ${ }^{[25]}$ proposed the use of E-rings which has fluoride releasing capabilities. The results were temporary due to the fluoride releasing capabilities of E-rings was lost by two weeks and bacterial colonies increased later.

According to the results based on Pellegrini et al ${ }^{[11]}$ where elastomeric rings on conventional brackets (GAC International) and self-ligating brackets (GAC International) were evaluated; and the results were like Erings had greater retention to the biofilm than self-ligating brackets, where the present study analysed 3 techniques of ligation with respect to biofilm retention. It is hypothesized that by the absence of any ligatures a self-ligation technique would be equal or more or less the same hygiene wise, when compared to ligature wires.

Based on the results given byPandiset al ${ }^{[17]}$ it can be taken that there were no statistical significant difference between conventional and self-ligating brackets in the levels of S.mutansin saliva of patients undergoing orthodontic treatment.

Van Gastel et al ${ }^{[12]}$ evaluated the metal self-ligating brackets Damon (Ormco) and Speed (Speed, canada), conventional brackets Victory series (3M Unitek) and Microloc (GAC). The results found by the author are that there were no statistical significant difference in bacterial counts between the metallic brackets groups of self-ligating brackets and conventional brackets.

Gracez et al ${ }^{[13]}$ presented a study evaluating the biofilm adhesion to orthodontic brackets by 3 methods of ligation and used OCT images to confirm them along with microbiologic sampling. The results obtained were similar to the present study.

Pithon et al ${ }^{[26]}$ documented a study on microbial adhesion on different self-ligating brackets. Their study revealed that Smart Clip brackets had the maximum amount of bacterial adhesion to their winglets, followed by Aditek, GAC, Ormco and conventional. When the channals were compared the Ormco brackets had the greatest bacterial adhesion and least to conventional system. In the cervical portion the Aditek formed the most colonies followed by GAC. Even though so many efforts and studies have been done, there is still noproper evidence that self-ligating brackets has decreased amount of plaque adhesion.

In the present study the biofilm retention to different parts of orthodontic brackets by three different methods of ligation were assessed and it was found that elastomeric module had higher retention of biofilm when compared to self-ligating brackets and steel ligatures. 
It can be said that self-ligating brackets have greater biofilm retention due to presence of clips, hinges and other retention areas in the bracket design when compared to steel wire ligatures which has least biofilm retention. The biofilm retention is found to be greatest in elastomeric module because they are polyurethanes, thermosetting polymer products which are not inert materials and are affected by heat, moisture and contact with salivary and oral enzymes. Water acts as a plastisizer of these materials by weakening the intermolecular forces, leading to chemical degradation. Elastomeric modules which are stretched and placed onto the brackets have a tendency to imbibe moisture and enlarge to which the bacteria are attracted.

\section{Conclusion}

The following conclusions can be drawn from the present study:

1. Fixed orthodontic appliances significantly increase the retention of biofilm (S.mutans MTCC 890) regardless the type of bracket system chosen (conventional or SLB).

2. The result of the present study indicates that steel ligature (Group-B) had least amount of biofilm retention when compared to elastomeric module (Group-A) and self-ligating bracket (Group-C).

3. In this study steel ligature group gain more clinical significance in orthodontic multi-bracket appliance therapy because of fewer areas for microbial shelters which may render the patients to low risk for dental caries and enamel demineralization.

Furthermore, increase in sample size and in-vivo studies are required to compare the result of this study to gain clinical significance.

\section{References}

[1] Scheie AA, Arneberg, Krogstad O: Effects of orthodontic treatment on prevalence of Streptococcus mutans in plaque and saliva. Scand. J. Dent. Res. 92 (1984)

[2] Lundstrom F and KrasseB : Caries incidence in orthodontic patients with high levels of streptococcus mutans. Eur J Orthod (1987) 9 (1): $117-121$.

[3] Richard G. Rosenbloom and Norman Tinanoff. Salivary S. mutans levels in patients before, during and after orthodontic treatment. Am J OrthodDentofacOrthop 1991;100:35-7.

[4] Eliades, T., Eliades, G., Dr. Odont, Brantley, W.A. Microbial Attatchment on orthodontic appliances: I. Wettability and early pellicle formation on bracketmaterials. Am J OrthodDentofacialOrthop; 108 (1995): 351-360.

[5] Fournier A., Payant L., Bouclin R.: Adherence of Streptococcus mutans to orthodontic brackets. Am J. of Orthodontics and DentofacialOrthop; Vol. 114:4 (1998): 414-417.

[6] Turkkahraman H, Sayin MO, Bozkurt FY, Yetkin Z, Kaya S, Onal S. Archwire ligation techniques, microbial colonization, and periodontal status in orthodontically treated patients." Angle Orthod 2005; 75: 231-6.

[7] Ahn et al. Quantitative determination of adhesion patterns of cariogenic streptococci to various orthodontic brackets. Am J OrthodDentofacialOrthop 2007; 132:815-21.

[8] Papaioannoua et al. Adhesion of Streptococcus mutansto Different Types of Brackets. Angle Orthodontist, Vol 77, No 6, 2007.

[9] Souza et al. Periodontal and microbiologic evaluation of 2 methods of archwire ligation: Ligature wires and elastomeric rings. Am J OrthodDentofacialOrthop 2008; 134: 506-12.

[10] Faltermeier et al. Bacterial adhesion of Streptococcus mutansto esthetic bracket materials. Am J OrthodDentofacialOrthop 2008;133: S99-103.

[11] Pellegrini et al. Plaque retention by self-ligation vs elastomeric orthodontic brackets: quantitative comparison or oral bacteria detection with adenosine triphosphate-driven bioluminescence. Am J OrethodDentofacialOrthop 2009; 135: 426.e1-9: discussion, 426-7.

[12] Van Gastel et al. Microbial Adhesion on Different Bracket Types in vitro. Angle Orthod. 2009; 79: $915-921$.

[13] Garcez et al. Biofilm retention by 3 methods of ligation on orthodontic brackets: A microbiologic and optical coherence tomography analysis. Am J OrthodDentofacialOrthop 2011; 140:e193-e198.

[14] Luca Lombardo, YildizÖztürkOrtan, ÖzgeGorgun, Chiara Panza, Giuseppe Scuzzo and Giuseppe Siciliani. Changes in the oral environment after placement of lingual and labial orthodontic appliances. Progress in Orthodontics 2013, 14:28

[15] Hayder F. Saloom ,Harraa S. Mohammed-Salih , Shaymaa F. Rasheed. The influence of different types of fixed orthodontic appliance on the growth and adherence of microorganisms (in vitro study). J ClinExp Dent. 2013;5(1):e36-41.

[16] Forsberg et al. Ligature wires and elastomeric rings: two methods of ligation, and their association with microbial colonization of Streptococcus mutans and lactobacilli. Eur J Orthod 1991; 13:416-20.

[17] Graber T.M, Vanarsdall R.L: Orthodontic Current Principles \& Techniques, 3rd edition Mosby, 2009.

[18] Lindel et al. Comparative analysis of long-term biofilm formation on metal and ceramic brackets. Angle Orthod. 2011;81:907-914.

[19] JitendraBhagchandani, Ashish Kumar Singh, Praveen Mehrotra, Shashi Kumar H. C., Shruti Raj Varshney, Krati Raj Varshney. Microbial colonization around orthodontic ligature ties: An in vivo study. APOS Trends in Orthodontics | May 2013 | Vol 3 | Issue 3 p-72-77.

[20] PanduranganHarikrishnan, ThillaiSakuSubha, VaradharajanKavitha, ArumugamGnanamani. Microbial Adhesion on Orthodontic Ligating Materials: An in Vitro Assessment. Advances in Microbiology, 2013; 3: p- 108-114.

[21] Imtiaz Ahmed, Saif-ul-Haque, RozinaNazir. Carious lesions in patients undergoing orthodontic treatment. JPMA 2011;61: 1176-79.

[22] Pejda et al. Clinical and microbiological parameters in patients with self-ligating and conventional brackets during early phase of orthodontic treatment. Angle Orthod. 2012.

[23] Corbet JA et al. Comparison of Streptococcus mutans concentration in banded and non-banded orthodontic patients. J Dent Res 1981; 60: 1936-42.

[24] Sukontapatipark et al. Bacterial colonization associated with fixed orthodontic appliances. A scanning electron microscopy study. Eur J Orthod. 2001;23:475-484

[25] Wilson T G, Gregory : Clinical effectiveness of fluoride-releasing elastomers. Salivary Streptococcus mutans numbers. Am J OrthodDentofacOrthop 1995;107:293-7.

[26] Pithon et al. Do self-ligating brackets favor greater bacterial aggregation? Braz J Oral Sci. 2011; 10:208-212. 